\title{
Analysis of Age Invariant Face Recognition using QSVM-PCA
}

ASHUTOSH DHAMIJA ( $\nabla$ dhamija.ashutosh@gmail.com )

SRMUH: SRM University Haryana - Campus

\section{R.B DUBEY}

SRMUH: SRM University Haryana - Campus

\section{Research}

Keywords: Age-invariant face, recognition, feature, extraction, PCA, and, QSVM

Posted Date: December 14th, 2020

DOI: https://doi.org/10.21203/rs.3.rs-124138/v1

License: (c) (i) This work is licensed under a Creative Commons Attribution 4.0 International License. Read Full License 


\title{
Analysis of Age Invariant Face Recognition using QSVM -PCA
}

\author{
Ashutosh Dhamija \\ ECE department, \\ SRM University, Sonepat, \\ Haryana, India. \\ dhamija.ashutosh@gmail.com
}

\author{
R. B. Dubey \\ EEE department, \\ SRM University,Sonepat, \\ Haryana, India \\ rbdubey@ srmuniversity.ac.in
}

\begin{abstract}
Forage, face recognition is one of the most demanding field challenges, since aging affects the shape and structure of the face. Age invariant face recognition (AIFR) is a relatively new area in face recognition studies, which in real-world implementations recently gained considerable interest due to its huge potential and relevance. The AIFR, however, is still evolving and evolving, providing substantial potential for further study and progress inaccuracy. Major issues with the AIFR involve major variations in appearance, texture, and facial features and discrepancies in position and illumination. These problems restrict the AIFR systems developed and intensify identity recognition tasks. To address this problem, a new technique Quadratic Support Vector Machine- Principal Component Analysis (QSVM-PCA) is introduced. Experimental results suggest that our QSVM-PCA achieved better results especially when the age range is larger than other existing techniques of face-aging datasets of FGNET. The maximum accuracy achieved by demonstrated methodology is $98.87 \%$.
\end{abstract}

Keywords- Age-invariant face recognition, feature extraction, PCA and QSVM.

\section{Introduction}

Age invariant face recognition (AIFR) is a key element across several uses, including forensic biometric monitoring technologies and the identification of missing persons. The facial characteristics gradually change with different age patterns per human. That refers to personal DNA, personal health, lifestyle, etc. Extracting robust features representing aging facial descriptions is a challenging research concern, particularly where broad age differences between face images are considered [1-4]. A well-known model database FGNET is being investigated for AIFR. The problems posed in the facts can be outlined as follows [5]:

Every object has a low-image vector series of different ages. Which makes it challenging to learn because of the small number of training products per participant and the fact that each participant has their aging period.

0 Possessing, lighting, blurring, and distance from the sensor vary from one image to another.

0 The section of data between the ages of 0-2 years, where clear customized features of the face have not yet been identified. It is very difficult at this age to compare the face of this age (0-2) with the right elder faces of the same individual, except with human being eyes.

Presence of high age gaps in FGNET (age from 0 to 69 years) in particular.

The method is mainly known that facial aging is a dynamic process that influences both the function and the expression of the face. The key change of age growth is craniofacial development in the early stages of the face from childhood to adulthood. When people grow aged from young to elderly, the primary factor is skin aging due to differences in texture.

There are many explanations for finding facial recognition more complicated than other variants under age differences: (a). The progression of age through life is not straightforward progression as mentioned above; (b). Outcomes of aging are very special to many individuals because the origin of the development of age is often impossible to determine precisely. For example, young people who are elderly would tend to be somewhat different from those experiencing disabilities or diseases in their lives; (c). It is therefore impossible to achieve adequate testing data to examine the consequences of aging since it requires much more time and more effort. Aging data sets taken from photographs of varying age ranges can be more skewed than other versions. Finally, but not least, nearly all of the research work relating to age is centered on datasets in which each person has an actual number. Aging data sets generated from pictures at varying age groups may be more blurred than other versions. This renders it difficult to identify machines since much of the techniques now known still teach machines to learn from the knowledge of face appearances. It can look very distinctive for two individuals of the same actual generation. Finally, the method of learning or marking would be less successful. A variety of age-related experiments have been 
proposed in recent years, based on age and age-invariant facial recognition or monitoring assessments [6-19]. While the fundamental hypotheses and approaches have varying functions, they converge and relate broadly. Usually, these two methods can be split into two classes. First, generative approaches $[6,11,16,18]$ that create 2D or 3D generational models to correct facial images, indicating the age of facial images in the aging process. The second method is focused on discriminatory [17,19-22] models that use comprehensive facial characteristics and discriminatory approaches to learning to minimize the disparity between face photos taken during different age groups. Both age and simulator use standard approaches for age-invariant face recognition activities. Age forecasts and simulators concentrate primarily on information that is linked to age development, while age-invariant face recognition aims to identify information that is secure for the same person over decades. This major disparity encourages a modern way of distinguishing the face from the aspect of age and the variable of personality [14, 18, 23]. In [21-25] one of the early studies on facial recognition depicted a mask with its inner and interindividual variations. In a generative linear model, Probabilistic Linear Discriminant Regression (PLDA) [26] was used and an ideal latent identity variable was obtained iteratively using EM [27]. This strategy was also used for identification of an age-invariant face in[18] where internal difference was defined by age-relevant information, and identity awareness was the interdependent discrepancy. Again, an EM algorithm is used to simultaneously remove and classify all latent variables. Experimental experiments have also demonstrated that all existing methods are effective for this method. This principle was subsequently also used to model aging ears, though remaining invariant over time[14], by representing the aging layer as a linear combination of age-progress intervals. All these approaches generate an aged subspace and a subspace for identification using a single structure. This method, however, has a large demand for training data sets; because personality and aging data must be taught as comprehensively as practicable.

Unfortunately, the processing of appropriate data sets for age-invariant face recognition is a major obstacle. For the three most well-known data sets for this assignment, either the absence of samples of training (FGNET dataset[28]) or the lack of samples of long-term established learning trends (MORPH[29] dataset and CACD datasets). Worse, both past curriculum frameworks centered on real age markers that can be consistent with youth and facial features of the era concerned. This results in limited facial recognition performance for age gaps. One approach to resolving age gaps consists of researching the fundamental temporal dynamics $[6,31]$ and then utilizing numerous analyses to determine age characteristics [7, 32]. It has been shown that the application of an OLPP to an aged population of ages from 0 to 93 years produces reliable statistical findings in terms of age. With the interest in subjects relating to age, the subsequent tasks in the next 100 years have become extremely difficult. One of the main sources of age growth is to measure the appearance age for the ChaLearn data set[34] public for wild face images, identified by their appearance age. We establish a generative model based on the PLDA, close to the method of aging and self-identification [14,18,23].In contrast to the earlier literature, which discovered and developed the subspaces of aging and identification, it is one of the key sources of study of age development. In the same way, as for the approach of aging and self-identification [14,18,23], we are creating a generative model focused on the PLDA. Previous literature that simultaneously analyzed and extracted aging and identity subspaces to achieve a higher recognition rate. First of all, this approach refers to the issue of age-invariant face recognition and allows it possible to easily and reliably analyze aging findings. This scheme's by-product will encourage the sorting of old images, where identification labels are only needed. Any aging dataset with marks of presence will learn the characteristics of aging. A powerful fusion process focused on Canonical Correlation Analysis (CCA)[35], has been used to further enhance distinguishing and aged sub-spaces, and to further develop the underlying identification factors based on various characteristics. Extensive experiments on three separate aging datasets indicate that the system can greatly boost the accuracy of rank-1 identification with other state-of-the-art methods, particularly when faced with wide differences in age.

Face recognition will verify the identification of an individual by equalizing facial biometrics as against recognized faces in a database. The group of the face with established affinities should be space and the input face must be determined by affinity. Confirmation in the face is one thing and acknowledgment is the other. Authentication is an ongoing process for testing the affinity of an individual. The weeping phrase is often associated with an expanded connotational version. It is used for access management where users are incorporated characteristically. Face Empathy is tougher since the complete Security Matching List help is provided and user assistance is not planned. Many challenges in developing an effective facial recognition device require shifts in the stance and age of a motion lighting brain. Many approaches for illumination and/or posing invariant facial recognition have been mentioned. Aging is a guaranteed normal practice of a human being's life. There are three peculiar features of the aging series [35,36,37]:

Aging development is unmanageable and is gradual and permanent. 
Aging patterns are customized and has a particular aging sequence for each individual who in turn depends on factors like environment, diet, fitness etc.

Time-based changing of aging patterns.

The entirety of the paper is thus organized. Section 2 addresses the study of literature. Section 3 describes the approach presented Section 4 offers tentative results and observations in face recognition focused on multiple aging data sets with different age marks. The hypothesis and future analysis are clarified in Section 5.

\section{Survey of literature}

AIFR methods are categorized as generative, discriminative, and deep-learning as follows:

\section{a) Generative approaches}

Generative approaches seek to mimic the aging mechanism by producing a synthesized facial picture utilizing old images acquired before face recognition. Lanitis et al. [13] have built a 3D model of virtual aging based on the structure and intensity characteristics of a private database that achieve an RR of 68.5\%. Park et al.[4] employ a virtual structural and shape 3D aging model for FGNET and MORPH data sets with an RR of $37.4 \%$ and $79.8 \%$ respectively. These models are limited by irrational, stable parametric assumptions, while generative approaches might simulate age models [38].

\section{b) Discriminative approaches}

Discriminative approaches exclude the standard face features that mostly represent the aging phase. Ling et al.[39] used the gradient orientation pyramid (GOP) for classification purposes to define an aging process and used the support vector machine (SVM). Li et al. [16] used transform (SIFT)[40] and multivariate local binary pattern (MLBP)[41] as differential characteristics for age-invariant detection. Gong et al. [15] used the maximum entropy descriptor (MEFD) function which encrypted the facial images in a variety of discreet entropy codes. Li et al.[42] studied discrimination using an updated hidden factor analysis (HFA). They found an age-gender interaction, instead of believing that they were equally distinct. Zhou et al . recently used an AIFR identity inference model based on linear probabilistic analytics and the EM algorithm[43].

\section{c) Convolutional neural networks (CNN)}

To overcome spatial associations in natural images, CNN uses fully-linked hidden layers and locally bound convolution layers, shared parameters, and detached parameters to significantly reduce the number of features CNN has learned[1]. The present research has shown that the performance of CNN layers generates extremely biassed AIFR descriptors. Using CNN to remove facial gestures, Yan et al.[44] used the SVM classification to use age. In comparison, Li et al. used a profound CNN model [45] that conducted both extraction and classification functions. $\mathrm{Xu}$ et al. [46] have used a related auto-encoder network to obtain the AIFR face register name. Li et al. [47] implemented a paradigm for optimizing AIFR functions and distance metrics at the same time. Shakeel et al [48] also used an innovative CNN design to strip facial characteristics. The extracted characteristics have been further encoded using the analyzed codebook and a linear encoding of regression to fit the face. Recently the pre-trained model VGG-Face CNN is commonly used in applications of facial recognition in $[49,1,50]$.

\section{Demonstrated Methodology}

This section describes an overview of demonstrated AIFR methodology and details of each process. 


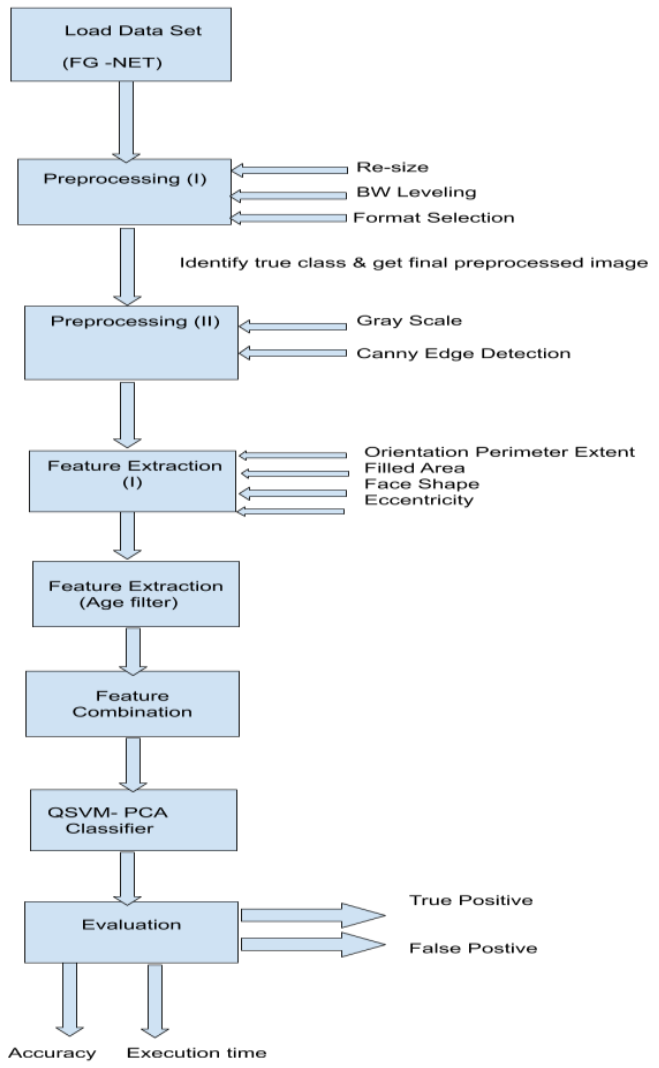

Fig. 1: Demonstrated Methodology

\section{Preprocessing}

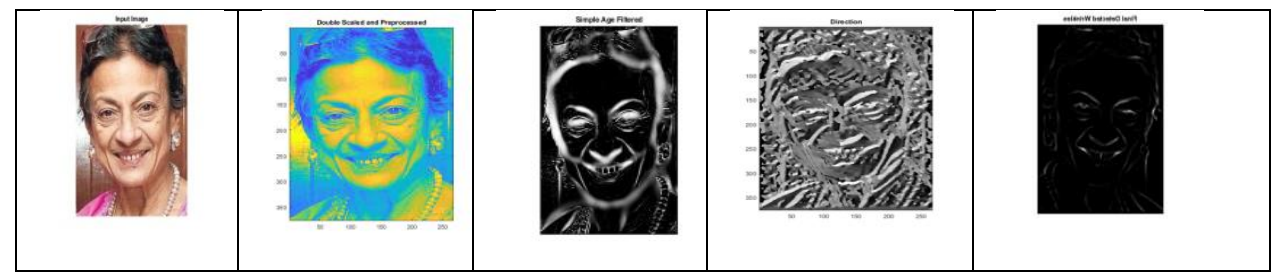

Fig. 2: Sample image of FGNET dataset.

Relevant images include image areas that distort face recognition, i.e. hair and clothing. In this way, we remove from the nose of the eyes and mouth of the particular picture a near box of the local face given in advance. Around the same moment, we uniformly alter the layout of the labeling and modify the grayscale.

\section{Feature extraction}

Part 1: Canny edge filter

A multi-stage edge detector is a Canny filter. It uses a filter dependent on a logarithmic derivative to measure the gradient strength. The logarithmic reduces the noise effect of the image. It is used to extract useful structural information from various facial images and to dramatically reduce pixels. It is a method of seeking out the rims by isolating image noise without impacting the characteristics of the rims and then using the inclination to locate the rims and the critical threshold value. The next steps [51-53] are: 
i) Converting image to grayscale: RGB image is translated to gray scale in this stage.

ii) Smoothing image: A next step in eliminating noise is image smoothing. For each orientation, the gradient is the first level of image derivatives. The gradient can be determined via a central difference in the preparation of the image. Gaussian blur filtering is used to smooth the image. To extract high-frequency noises from the image, you obtain the image with a logarithmic clip.

iii) Image gradient: The gradient is part of the variables' function. To accomplish this partial derivative vertical and horizontal axis of the image convolution approach is performed with a Sobel filter.

iv) Non-maximum suppression: This step decides whether or not such a point is a neighborhood limit with an interjected pixel tendency, and this step influences the edge view greatly. This parameter is typically not particularly helpful to set the parameter to zero.

v) Tracking edge by hysteresis: We choose two styles of thresholds at this level, high and low. Each pixel compares two distinctive thresholds. In the last image, this pixel is marked 255 if the pixel is greater than the high threshold. May the pixel be smaller than the dark color of the lower threshold picture with 0 values in images.

Part 2: Feature extraction and creation of age filter

This section enhanced Active Shape Models (ASM), developed specifically to decide the facial age based on the shape of the subject. In conjunction with the age filtration system, the shape model provides a special estimate of face age, which helps to assess the face structure and produces a hybrid AE model. For more enhancement of accuracy, segmented facial sections are used.

Extraction of Global Image features

Attributes may be collected to obtain information on facial characteristics as global attribute descriptors. Shape measurement is a direct calculation of the appearance of a person. The list of numbers and perimeter features of Euler is extracted here.

\section{Combination of features and dimensionality reduction}

There are many features collected from dataset. Here, dimensionality reduction approach PCA is used to map high dimensionality feature space to low high variance subspace.

\section{PCA algorithm for feature extraction}

One of the most effective methods for image identification and compression is the Principal Component Analysis ( PCA). The reason for using PCA for FR is that large 1-D vectors of 2-D face pixels are voiced in smaller PCAs in the component region. This is known to be a different space projection. A decent threshold is usually difficult to select[54].

Offer a face an opportunity to view an $\mathrm{M}$ of two-dimensional values by $\mathrm{N}$ series. A range of 200 pixels per 149 pixels is used here. An image can also be used as a measurement vector $\mathrm{M}$ by $\mathrm{N}$, with the intention of turning the ordinary image of AI in the size of 200 x 149 into a measurement vector, 29,800 or a dot similarly in the size of 29 , 800 .

Step1: Preparation training faces to obtain face images $I_{1}, I_{2}, I_{3} \ldots I_{M}$ (training faces). The face images must be centered and of same size.

Step 2: Prepare data set each face image $I_{1}$ in database is transformed into a vector and placed into a training set $\mathrm{S}$.

$\mathrm{S}=\left\{\tau_{1}, \tau_{2} \tau_{3}, \ldots \ldots \ldots \ldots \ldots . \tau_{M}\right\}$

This is $\mathrm{M}=34$. Each picture is translated to an $\mathrm{MN} \times 1$ size vector and mounted.

Step 3: Computation of average face vector $(\Psi)$ is done by:

$\Psi=\frac{1}{M} \sum_{n=1}^{M} \tau_{M}$ 
Step 4: The average face vector $\Psi$ is subtracted from original face $s \tau_{i}$ and the result stored in the variable $\emptyset_{i}$.

$\emptyset_{i}=\tau_{i}-\Psi$

Step 5: Matrix $\mathrm{C}$ of covariance as determined

$\mathrm{C}=\frac{1}{M} \sum_{n=1}^{N} \emptyset_{N} \emptyset_{N}^{T}$

$=\mathrm{A} A^{T} \quad\left(N^{2} \times N^{2} \quad\right.$ matrix $) \quad$ where

$\mathrm{A}=\left[\emptyset_{1}, \emptyset_{2}, \emptyset_{3}, \emptyset_{4} \ldots \ldots \ldots \ldots \ldots . \emptyset_{M}\right]$

Step 6: Determine the eigenvectors and eigenvalues of the covariance matrix. The covariance matrix $\mathrm{C}$ in step 5 has a dimensionality of consequently individual would contain eigenface and eigenvalues. For a $256 \times 256$ images with the intention of means to the individual should compute a $65,536 \times 65,536$ matrix and compute 65,536 eigenfaces. Computationally this is not much competition as mainly of an individual's eigenfaces be not helpful designed for the task. So, compute the eigenvectors $u_{i}$ of $\mathrm{A} A^{T}$

The matrix $\mathrm{A} A^{T}$ is very large.

Step 6.1: consider matrix $(\mathrm{M} \times \mathrm{M}$ matrix $)$

$\mathrm{L}=A^{T} \mathrm{~A} \quad(\mathrm{M} \times \mathrm{N}$ matrix $)$

Step 6.2: compute eigenvectors

Step 6.3: compute eigenvectors $v_{i}$ of $\mathrm{L}=A^{T} A$

$A^{T} A v_{i}=\mu_{i} v_{i}$

$\mathrm{A} A^{T} A v_{i}=\mu_{i} \mathrm{~A} v_{i}$

$\mathrm{C} A v_{i}=\mu_{i} \mathrm{~A} v_{i}\left[\mathrm{C}=\mathrm{A} A^{T}\right]$

$\mathrm{C} u_{i}=\mu_{i} \mathrm{~A} v_{i} \quad$ where $u_{i}=A v_{i}$

Thus $\mathrm{C}=\mathrm{A} A^{T}$ and $\mathrm{L}=A^{T} A$ have same eigen values and their eigenvectors are related as follows:

$u_{i}=A v_{i}$

Step 7: Hold just K vectors (related to K's greatest own values). Eigenfaces with low eigenvalues are excluded, as they clearly only a small part of the features of the faces.

\section{Quadratic kernel-free non-linear SVM}

To solve quadratic problems, a new quadratic kernel-free nonlinear vector support (QSVM) is used. There is no need to use dual kernel trick optimization. A quadratic function (W bc) that can split non-linear data into two groups is indicated

$f(X)=\frac{1}{2} X^{T} W X+b^{T} \mathrm{X}+\mathrm{C}$

wherever $\mathrm{W}=[\mathrm{W} 1, \mathrm{~W} 2 \ldots \ldots \ldots \ldots \mathrm{Wm}]$ and $\mathrm{b}$ is a scalar.

$\mathrm{W}=W^{T}\left[\begin{array}{cccccc}W 11 & W 21 & \cdot & \cdot & \cdot & W 1 m \\ W 12 & W 22 & \cdot & \cdot & \cdot & W 2 m \\ \cdot & \cdot & \cdot & \cdot & \cdot & \cdot \\ \cdot & \cdot & \cdot & \cdot & \cdot & \cdot \\ \cdot & \cdot & \cdot & \cdot & \cdot & \cdot \\ W 1 M & W 2 M & \cdot & \cdot & \cdot & W m m\end{array}\right] ; \quad \mathrm{b}=\left[\begin{array}{c}b 1 \\ b 2 \\ \cdot \\ \cdot \\ \cdot \\ b m\end{array}\right] \ldots \ldots(2)$

It is assumed that: a) the decision surfaces $f(X)=$ ct can be of wide-ranging forms of hyper-planes, hyper-spheres, hyper-ellipsoids, hyper-paraboloids, hyper-hyperboloids of different type, and b) $f(X)$ is consider as the sum of two conditions: the non-linear term $\left(f_{\text {non-linear }}(X)=\frac{1}{2} X^{T} W X\right)$ and the linear term $\left(f_{\text {linear }}(X)=b^{T} \mathrm{X}+\mathrm{C}\right) \cdot[55]$

Pseudo Code for QSVM

Input: Training Sample with labels $T=\{\vec{x}, y\}$ where $\vec{x} \in \Omega C R^{n}$ and $y \in C$.

Parameters: $\vec{\theta}$ Initial parameter 
While $R_{\text {emp }}(\vec{\theta})$ has not converged do

for $i=1$ to $|T|$ do

setr $_{y}=0$ for every $y \in C$

* here $r_{y}$ is a counter

For shot $=1$ to $R$ do

prepare initial feature map state $\left|\emptyset\left(\vec{x}_{i}\right)><\emptyset\left(\vec{x}_{i}\right)\right|$ by using $U_{\emptyset\left(x_{i}\right)}$

apply discriminator circuit $W(\vec{\theta})$ to initial feature map state.

get outcome measurement $\left\{M_{y}\right\}_{y \in C}$ by appling $|C|$

get measurement outcome label y by setting $r_{y} \rightarrow r_{y}+1$

end

Calculate empirical distribution $\hat{p}_{y}\left(\vec{x}_{i}\right)=r_{y} R^{-1}$

Check the accuracy and error rate by evaluating

$P_{r}\left(\left(\breve{m}\left(\vec{x}_{i}\right) \neq y_{i} \mid m(\vec{x})=y_{i}\right)\right)$ with $\hat{p}_{y}\left(\vec{x}_{4}\right)$ and $y_{i}$

end

end

\section{Experimental results and analysis}

In specific, the FGNET dataset[56] MORPH dataset[57], and CACD data set[58] are compared with other best techniques. FGNET is called the largest facial maturation dataset and has also been used to perform facial expression-related age studies. The MORPH data set comprises two parts, MORPH one and MORPH two sets. Since collection one is limited (only 1690 pictures) latest collection two have been used for study, as the set contains 55,134 face images of 13,617 individuals. The newest maturing data set is CACD, which includes 163,446 images of 2000 esteemed web-based individuals. Any face images are reviewed and tested as below. The FGNET measures only data sets because it contains the smallest number of images, but the highest age difference.

Each of our parameters is chosen from past works and our test findings for an assessment of our model in depth. Maybe the most important leeway in our approach is that the names for planning tasks in age trials are never needed again because we have taken autonomously a maturing sub-space to the induction model of personality. Besides the FGNET dataset, the overall images are 1002 while the number of elements is even greater. We also related an important way of coping with the problem of exercise. Unlike previous systems [59, 60] that connect irregular undercut spaces with highlight cutting, ChaLearn and FGNET images use images with $95 \%$ of the fluctuation in the subspace of PCA. More DAM strength can be safeguarded, and the maturing images from ChaLearn dataset can also boost mature example learning, expecting an analogous subspace in PCA. Our estimation is tweakened by the FGNET model of data set which is prepared using FGNET, which also can be related to recognize the faces of various displays of data sets. We also carried out a detailed review and connation of some of the better current AIFR techniques. 240 FGNET images are transformed by $80 \mathrm{sec}$. The true PR appears in figure 4 . The cumulative iteration to be done is 1 for 1400 iterations..

\begin{tabular}{|c|c|c|c|c|c|c|c|c|c|}
\hline $\begin{array}{l}\text { Sr. } \\
\text { No. }\end{array}$ & Image 1 & Image 2 & $\begin{array}{l}\text { Image } \\
3\end{array}$ & Image 4 & Image 5 & Image 6 & Image 7 & $\begin{array}{l}\text { Image } \\
8\end{array}$ & Image 9 \\
\hline 1 & & & & & & & & & \\
\hline 2 & & & & & & & & & \\
\hline & & & & & & & & & \\
\hline & & & & & & & & & \\
\hline
\end{tabular}




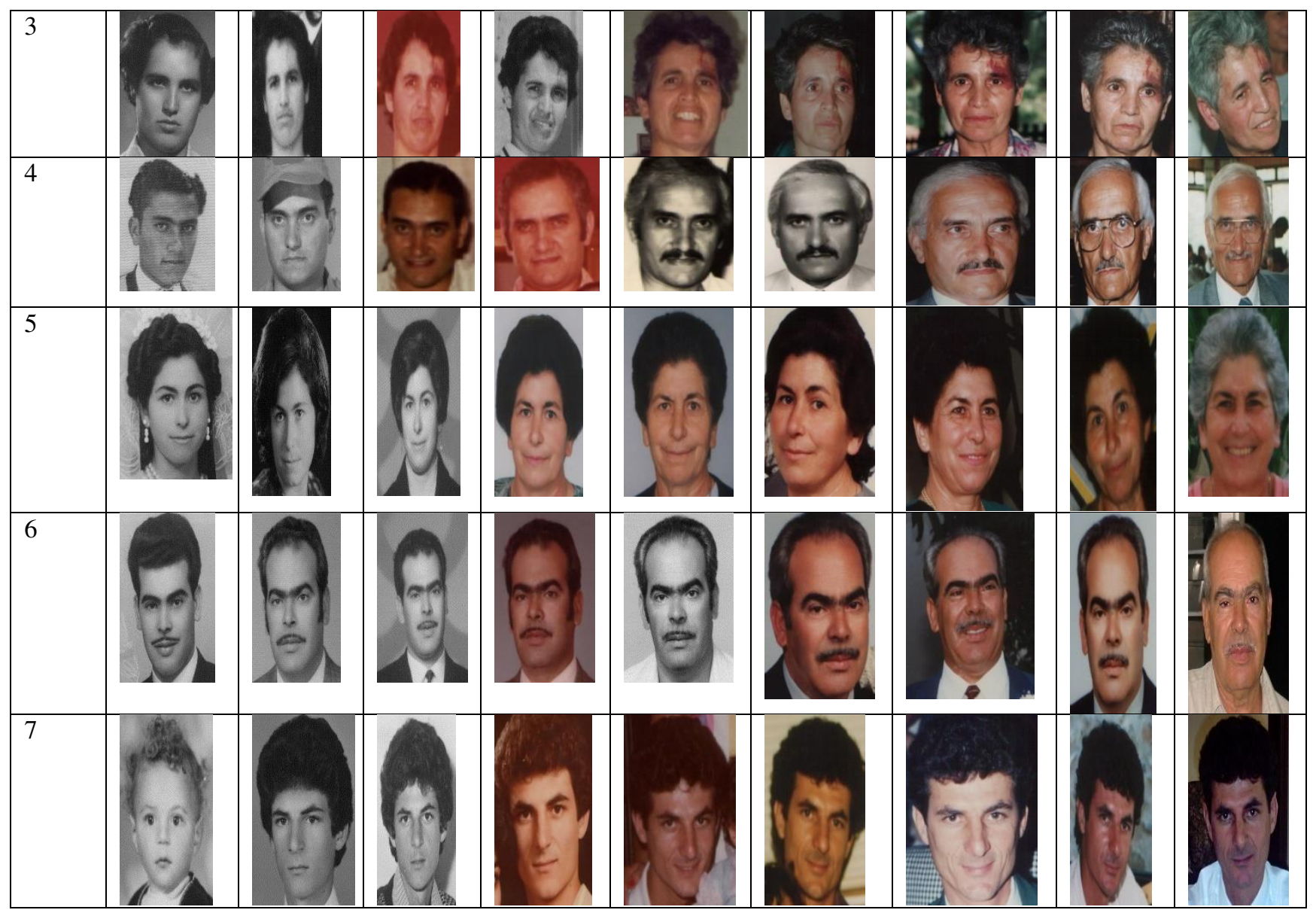

Fig.3: Sample images from FGNET database.

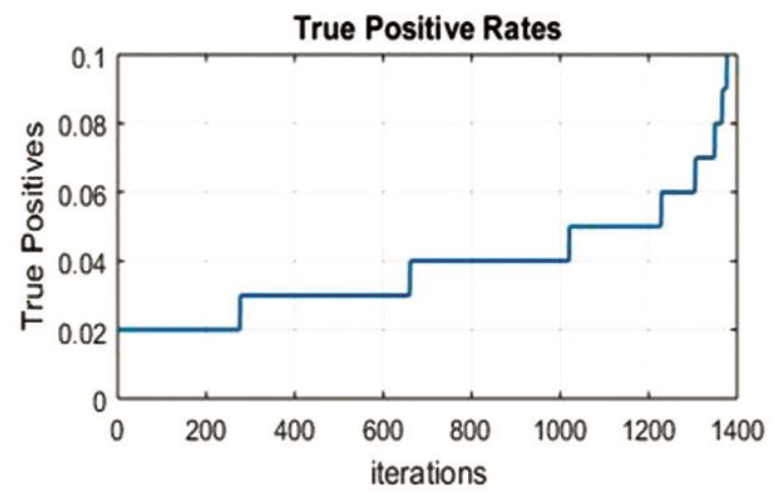

Fig 4: True PR (PR) 


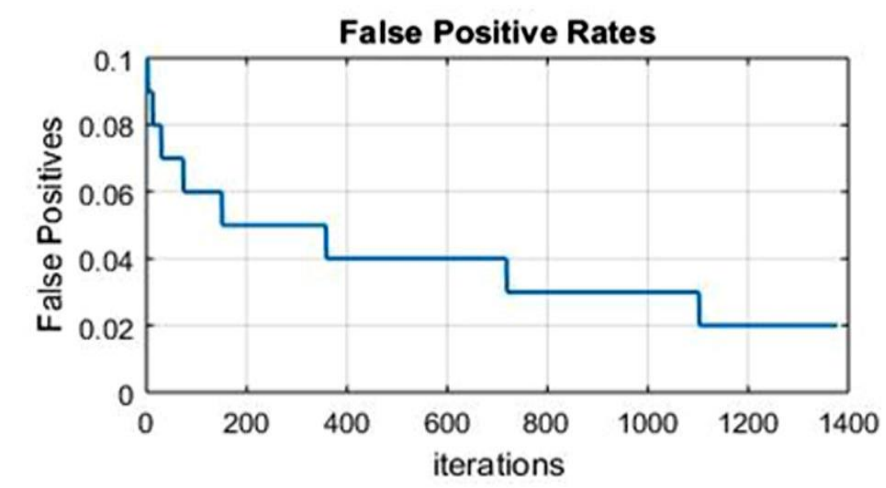

Fig. 5: False PR

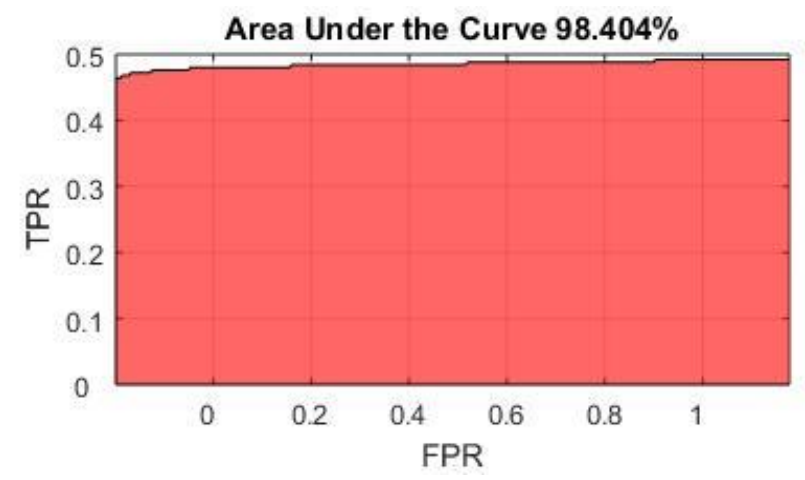

Fig. 6: Comparison of True Positive \& False Positive

By Calculation formula :

Precision : $\mathrm{P}=\mathrm{TP} /(\mathrm{TP}+\mathrm{FP})$,

Recall : $\mathrm{R}=\mathrm{TP} /(\mathrm{TP}+\mathrm{FN})$,

F1-score : 2/ (1/P+1/R),

ROC/AUC : TPR=TP/ (TP+FN),

$\mathrm{FPR}=\mathrm{FP} /(\mathrm{FP}+\mathrm{TN})$

ROC / AUC are same criteria and PR (Precision-Recall) curve (F1-score, Precision, Recall) is also same criteria. Real data will face imbalance problem, namely imbalance between positive and negative samples.ROC/AUC curve can remain curve, but PR change intensely when testing set occurs imbalance. The sum of true PR and false PR is 1. False PR is shown in Fig. 5. Comparison of True PR and False $\mathrm{PR}$ is shown in Fig. 6. Comparative analysis is given 0.5 curve area.

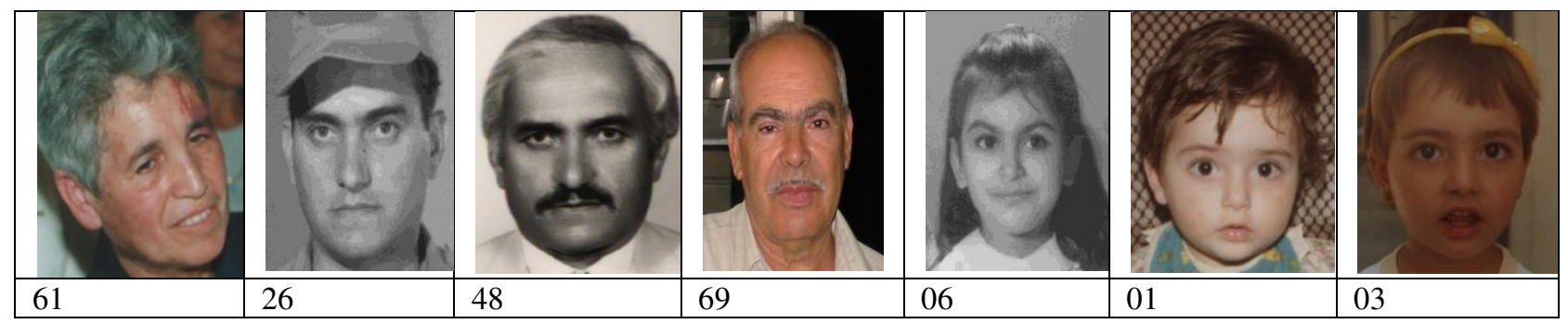




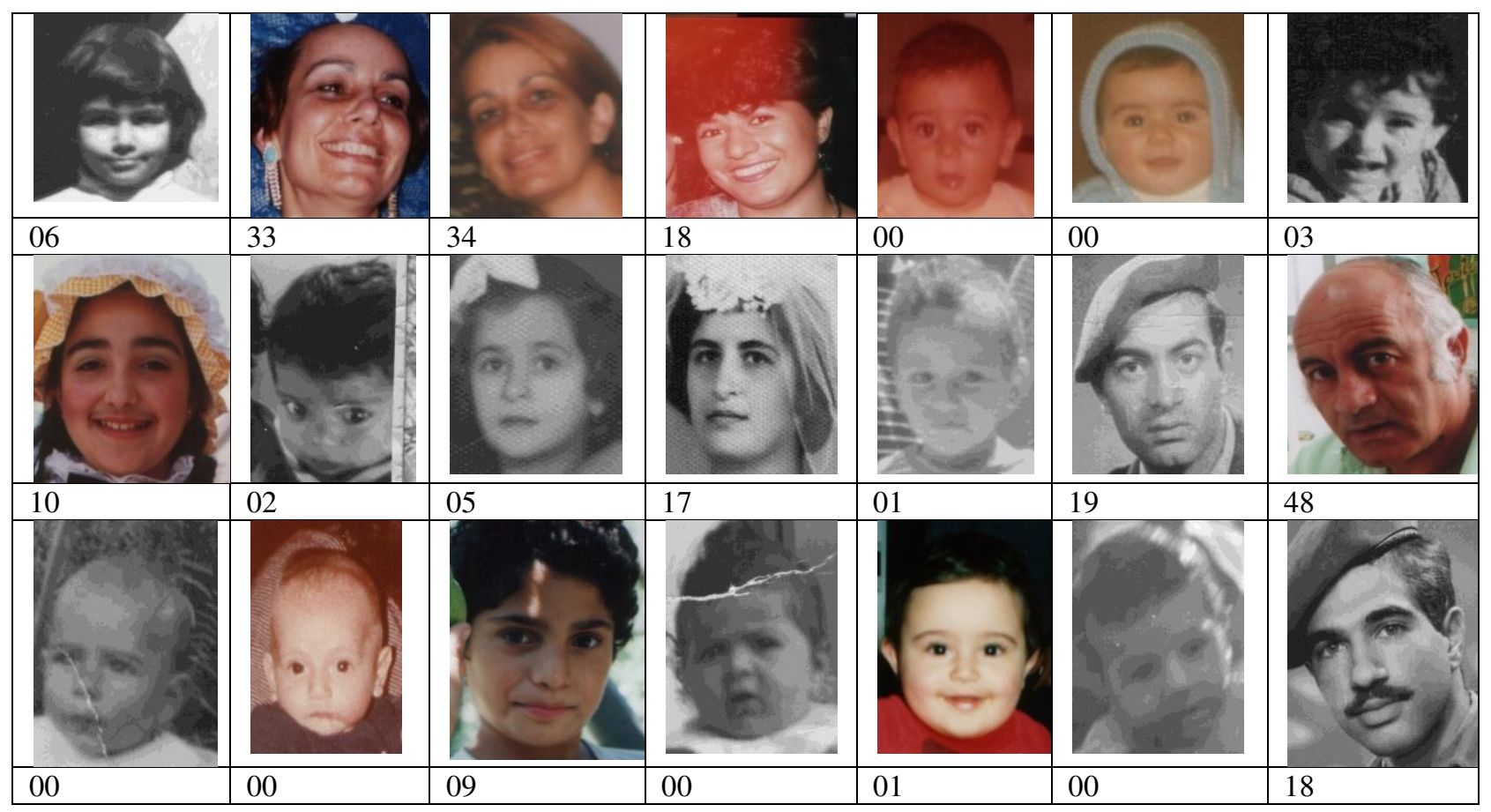

Fig 7: Some findings were incorrectly defined by the proposed FGNET dataset process. The numbers below display the age of the person.

Total images in FGNET Dataset 1002 images and 28 images incorrectly recognized results using proposed method.

Table 1: Comparative analyses of different methods and demonstrated method

\begin{tabular}{|l|l|l|}
\hline Sr. No. & Method & Accuracy \\
\hline 1 & 3D aging (2010) [4] & $37.4 \%$ \\
\hline 2 & Discriminative aging (2011) [16] & $47.5 \%$ \\
\hline 3 & Hidden factor analysis (2013) [17] & $69.0 \%$ \\
\hline 4 & Feature-aging (2015) [61] & $71.3 \%$ \\
\hline 5 & Maximum Entropy (2015) [15] & $76.2 \%$ \\
\hline 6 & AG -IIM with MLBP Feature (2018) [33] & $80.8 \%$ \\
\hline 7 & AG -IIM with HOG Feature (2018) [33] & $84.14 \%$ \\
\hline 8 & AG-IIM with CCA Feature Fusion (2018) [33] & $88.23 \%$ \\
\hline 9 & Demonstrated QSVM-PCA & $98.87 \%$ \\
\hline
\end{tabular}

Table 1 provides a comparative study of multiple techniques such as features AM, HFA, FG, ME, and AG-IIM. Table 1 contained an overview of performance as opposed to QSVMPCA and a range of advanced FGNET AIFR techniques like 3D maturing [4], DAM[16], FA [61], ME [15], and AG-IIM technology [33]. Our model is based on FGNET's data sets, while most of the AIFR models in [62] are compared to a few other facial data sets. Exhibition changes can hardly be understood from the technique itself or large-scale knowledge planning for varying training scales. In comparison with other first quality approaches designed entirely on FGNET, our approach produces oriented efficiency. Our model executes recognition accuracy and run time for FGNET 240 images 98.87 percent and $80 \mathrm{sec}$ separately. 


\section{Conclusions}

Age-invariant face recognition (AIFR) is a relatively new field of face-recognition science that has recently gained tremendous popularity owing to its immense ability and relevance in real-world applications. However, the AIFR is still in the phases of emergence and growth, providing a wide space to further investigate and enhance accuracy. We implemented a new QSVM-PCA technique that packs an enormous high-dimensional dataset by decreasing PCA-dependent dimensionality.

Experiments prove that our algorithm will take control of the scourge of dimensionality to address certain functional issues. The execution period of 240 FGNET images is $80 \mathrm{sec}$ and the accuracy obtained is 98.87 percent. Our future studies will focus on more age-invariant face recognition improvements utilizing Convolutional Neural Network in combination with an active shape model on more datasets.

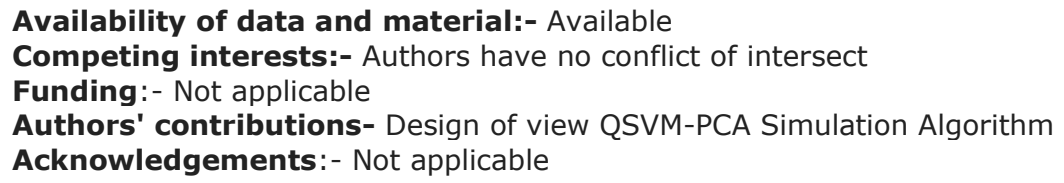

\section{References}

[1] H. E. Khiyari and H. Wechsler. Face recognition across time lapse using convolutional neural networks. Journal of Information Security, vol. 7, no. 03, pp. 141- 151, 2016.

[2] A. K. Jain, B. Klare and U. Park. Face matching and retrieval in forensics applications. IEEE Multimedia. vol.19, no.1, pp. 1-9, 2012.

[3] A. K.Jain and S.Z. Li. Handbook of Face Recognition. Springer, New York (2011).

[4] U. Park, Y. Tong and A. K. Jain. Age-invariant face recognition. IEEE Transactions on Pattern Analysis and Machine Intelligence (PAMI), vol. 32, no.5, pp. 947-954, 2010.

[5] Face group, G.R.W., et al:: Fg-Net Aging Database, 2000.

[6] X. Geng, H-Z. Zhou and K-M Smith. Automatic age estimation based on facial aging patterns. IEEE Transactions on Pattern Analysis and Machine Intelligence, vol. 29, no.12, pp. 2234-2240 ,2007.

[7] Y. Fu and T.S. Huang. Human age estimation with regression on discriminative aging manifold, IEEE Transaction on Multimedia. vol. 10, no. 4,pp. 578-584, 2008 .

[8] G. Mu , G. Guo , Y. Fu and T.S. Huang. Human age estimation using bio-inspired features, IEEE Conference on Computer Vision and Pattern Recognition, pp. 112-119, 2009.

[9] A. Montillo and H. Ling. Age regression from faces using random forests, IEEE International Conference on Image Processing, pp. 2465-2468, 2009.

[10] K. Zhu , D. Gong , Z. Li and X. Tang. Orthogonal gaussian process for automatic age estimation. International Conference on Multimedia, pp. 857-860, 2014.

[11] A. Lanitis , C.J. Taylor and T.F. Cootes. Toward automatic simulation of aging effects on face images, IEEE Transaction on Pattern Analysis Machine. Intelligence, vol. 24, no. 4,pp. 442-455, 2002

[12] J. Suo , S.C. Zhu , S. Shan and X. Chen. A compositional and dynamic model for face aging, IEEE Transaction on Pattern Analysis Machine. Intelligence ,vol. 32, no. 3 , pp. 385-401, 2010.

[13] J. Suo , X. Chen, S. Shan and W. Gao . Learning long term face aging patterns from partially dense aging databases. IEEE Conference on Computer Vision, pp. 622-629, 2009.

[14] X. Shu , J. Tang , H. Lai , L. Liu and S. Yan. Personalized age progression with aging dictionary. IEEE Conference on Computer Vision, pp. 3970-3978, 2015.

[15] D. Gong, Z. Li, D. Tao, J. Liu, and X. Li. A maximum entropy feature descriptor for age invariant face recognition. IEEE Conference on Computer Vision and Pattern Recognition (CVPR), pp. 52895297, 2015.

[16] Z. Li, U. Park U and AK. Jain. A discriminative model for age invariant face recognition. IEEE Transactions on Information Forensics and Security vol.6, no.3, pp. 1028-1037, 2011. 
[17] D. Gong, Z. Li, D. Lin, J. Liu and X. Tang. Hidden factor analysis for age invariant face recognition. IEEE International Conference on Computer Vision (ICCV), pp. 2872-2879, 2013.

[18] B. Chen , C. Chen and W.H. Hsu. Cross-age reference coding for age-invariant face recognition and retrieval. European Conference on Computer Vision, pp. 768-783, 2014.

[19] H. Ling , S. Soatto , N. Ramanathan and D.W. Jacobs. Face verification across age progression using discriminative methods, IEEE Transaction on information forensience Security vol. 5, no. 1, pp. 82-91, 2010.

[20] C. Otto, H. Ha and A. K. Jain. How does aging affect facial components. European Conference on Computer Vision Workshops and Demonstrations, pp. 189- 198, 2012.

[21] L. Du and H. Ling. Cross-age face verification by coordinating with cross-face age verification. IEEE Conference on Computer vision and Pattern Recognition. pp. 2329-2338, 2015.

[22] S.J. Prince and J.H. Elder. Probabilistic linear discriminant analysis for inferences about identity. IEEE Conference on Computer Vision, pp. 1-8, 2007.

[23] S. Ioffe. Probabilistic linear discriminant analysis. IEEE International Conference, European Conference on Computer Vision, pp. 531-542, 2006.

[24] P. Li , Y. Fu , U. Mohammed , J.H. Elde and S.J. Prince. Probabilistic models for inference about identity, IEEE Transaction on Pattern Analysis on Machine Intelligence, vol. 34, no 1, pp. 144-157, 2012 .

[25] Y.J. Zhang . Advances in Face Image Analysis: Techniques and Technologies, IGI Global, 2010 .

[26] A. Dempster , N. Laird and D. Rubin. Maximum likelihood from incomplete data via the EM algorithm, J. R. Statistical Soc. pp.1-38,1977 .

[27] K. Ricanek and T. Tesafaye. Morph: A longitudinal image database of normal adult age-progression, in: IEEE Int. Conf. Automat. Face Gesture Recognition , pp. 341-345, 2006 .

[28] T. Cootes and A. Lanitis. FG-NET aging database [Online], 2008. Available http:// www.fgnet.rsunit.com/ .

[29] B. Chen , C.S. Chen and W. Hsu. Face recognition and retrieval using cross-age reference coding with cross-age celebrity dataset, IEEE Trans. Multimedia, vol 17, no 6, pp. 804-815, 2015.

[30] K.A. Deffenbacher , T. Vetter , J. Johanson and O. Toole. Facial aging, attractiveness, and distinctiveness, Perception, vol. 27, pp. 1233-1243, 1998.

[31] G. Guo , Y. Fu , C. Dyer and T.S. Huang. Image-based human age estimation by manifold learning and locally adjusted robust regression, IEEE Trans. Image Process. vol.17, no. 7, pp. 1178-1188, 2008

[32] D. Cai , X. He , J.W. Han and H. Zhang . Orthogonal laplacian faces for face recognition, IEEE Trans. Image Process. Vol. 15, no. 11, pp. 3608-3614, 2006.

[33] S. Escalera , M. Torres , B. Martinez , X. Baro , H.J. Escalante , I. Guyou , G. Tz- imiropoulos , C. Corneanu , M. Oliu , M.A. Bagheri and M. Valstar. ChaLearn looking at people and faces of the world: face analysis workshop and challenge. IEEE Conference on Computer Vision and Pattern Recognition Workshop, pp. 1-8, 2016.

[34] D.R. Hardoon , S. Szedmak and J. Shawe-Taylor. Canonical correlation analysis: an overview with application to learning methods. Neural Computing. vol. 16, no. 12, pp. 2639-2664, 2004.

[35] S.S. Aashmi and S. Saxena. Survey: Techniques for Aging Problems in Face Recognition. International Journal of Computer Science and Information Technology vol. 4, no. 2, pp. 82-88, 2014.

[36] A. A. Osman V. Sagayan, A. Saeed, H. Ameen and A. Aziz. Age-invariant face recognition system using combined shape and texture features. IET Biometrics, vol. 4, no. 2, pp. 98-115, 2015.

[37] N. S. Devi and K. Hemachandran. Automatic Face Recognition System using Pattern Recognition Techniques: A Survey. International Journal on Computer Application, vol. 83, no. 5, pp. 10-13,2013.

[38] D. Gong, Z. Li, D. Lin, J. Liu and X. Tang. Hidden factor analysis for age invariant face recognition. In: Proceedings of the IEEE International Conference on Computer Vision, pp. 2872-2879, 2013

[39] H. Ling, S. Soatto, N. Ramanathan and D. W. Jacobs. Face verification across age progression using discriminative methods. IEEE Transaction on Informatics Forensics Security. vol. 5, no. 1,pp. 82-91, 2010.

[40] Lindeberg, T.: Scale Invariant Feature Transform (2012).

[41] T. Ojala, M. Pietikainen and T. Maenpaa. Multiresolution gray-scale and rotation invariant texture classification with local binary patterns. IEEE Transaction on Pattern Analysis Machine. Intelligence, vol. 24, no. 7, pp. 971-987, 2002. 
[42] H. Li, H. Zou and H. Hu. Modified hidden factor analysis for cross age face recognition. IEEE Journal on Signal Processing Letters, vol.24, no.4, pp. 465-469,2017.

[43] H. Zhou and K.M. Lam .Age-invariant face recognition based on identity inference from appearance age. Pattern Recognition, vol. 76, pp.191-202, 2018.

[44] C. Yan, C. Lang, T. Wang, X. Du and C. Zhang . Age estimation based on convolutional neural network. In: Pacific Rim Conference on Multimedia, pp. 211-220, 2014.

[45] Y. Li, G. Wang, L. Lin and H. Chang. A deep joint learning approach for age invariant face verification. In: CCF Chinese Conference on Computer Vision, pp. 296-305, 2015.

[46] C. Xu, Q. Liu and M. Ye, Age invariant face recognition and retrieval by coupled auto-encoder networks. Neurocomputing, vol. 222, pp. 62-71, 2017.

[47] Li, Y, Wang, G., Nie, L.,Q. Wang and W. Tan. Distance metric optimization driven convolutional neural network for age invariant face recognition. Pattern Recognition. vol.75, pp. 51-62, 2018.

[48] Shakeel, M.S., Lam and K.M. Deep-feature encoding-based discriminative model for age-invariant face recognition. Pattern Recognition. vol. 93, pp. 442-457,2019.

[49] A. Anand, R. D. Labati, A. Genovese, E. Ballester, V. Piuri and F. Scotti. Age estimation based on face images and pretrained convolutional neural networks.IEEE Symposium Series on Computational Intelligence(SSCI), 2017.

[50] O. M . Parkhi, A. Vedaldi and A. Zisserman. Deep face recognition. In: BMVC, vol. 1, pp. 1$41,2015$.

[51] J.F. Canny. A computational approach to edge detection. IEEE Transactions on pattern analysis and machine intelligence vol. 6, pp.679-698, 1986.

[52] A. Ayanzadeh, H. Pourghaemi and Y. Seyfari. A modified ant colony based approach to digital image edge detection. IEEE International Conference on Knowledge-Based Engineering and Innovation (KBEI), 2015.

[53] Irandoust-Pakchin, S.,Ayanzadeh, A and Beikzadeh. Gaussian Three-Dimensional kernel SVM for Edge Detection Applications. arXiv preprint arXiv:1710.01260,2017.

[54] L. Sirovich and M Kirby. A low dimensional procedure for the characterization of human faces, vol. 4, no. 3, pp.519-524, 1987.

[55] Issam Dagher. Quadratic kernel-free non-linear support vector machine, Springer, J. Glob Optim, vol.41, pp. 15-30, 2008.

[56] B.-C. Chen, C.-S. Chen and W. H. Hsu. Face recognition and retrieval using cross-age reference coding with cross age celebrity dataset. IEEE Transactions on Multimedia, vol. 17, no. 6, pp.804-815, 2015.

[57] Z. Liu, P. Luo, X. Wang and X. Tang. Deep learning face attributes in the wild. International Conference on Computer Vision (ICCV), 2015.

[58] A. Nech and I. Kemelmacher-Shlizerman. Level playing field for million scale face recognition. IEEE Conference on Computer Vision and Pattern Recognition (CVPR), 2017.

[59] S. Ioffe and C. Szegedy. Batch normalization: Accelerating deep network training by reducing internal covariate shift. International Conference on Machine Learning, 2015.

[60] I. Kemelmacher-Shlizerman, S. M. Seitz, D. Miller and E. Brossard. The mega face benchmark: 1 million faces for recognition at scale. IEEE Conference on Computer Vision and Pattern Recognition (CVPR), 2016.

[61] H. Zhou , K. Wong and K. Lam . Feature-aging for age-invariant face recognition, IEEE AsiaPacific Signal and Information Processing Association Annual Summit and Conference (APSIPA), pp. 1161-1165, 2015.

[62] R. Rothe, R. Timofte and L. V. Gool.Dex: Deep expectation of apparent age from a single image. International Conference on Computer Vision Workshops (ICCVW), 2015. 
Figures

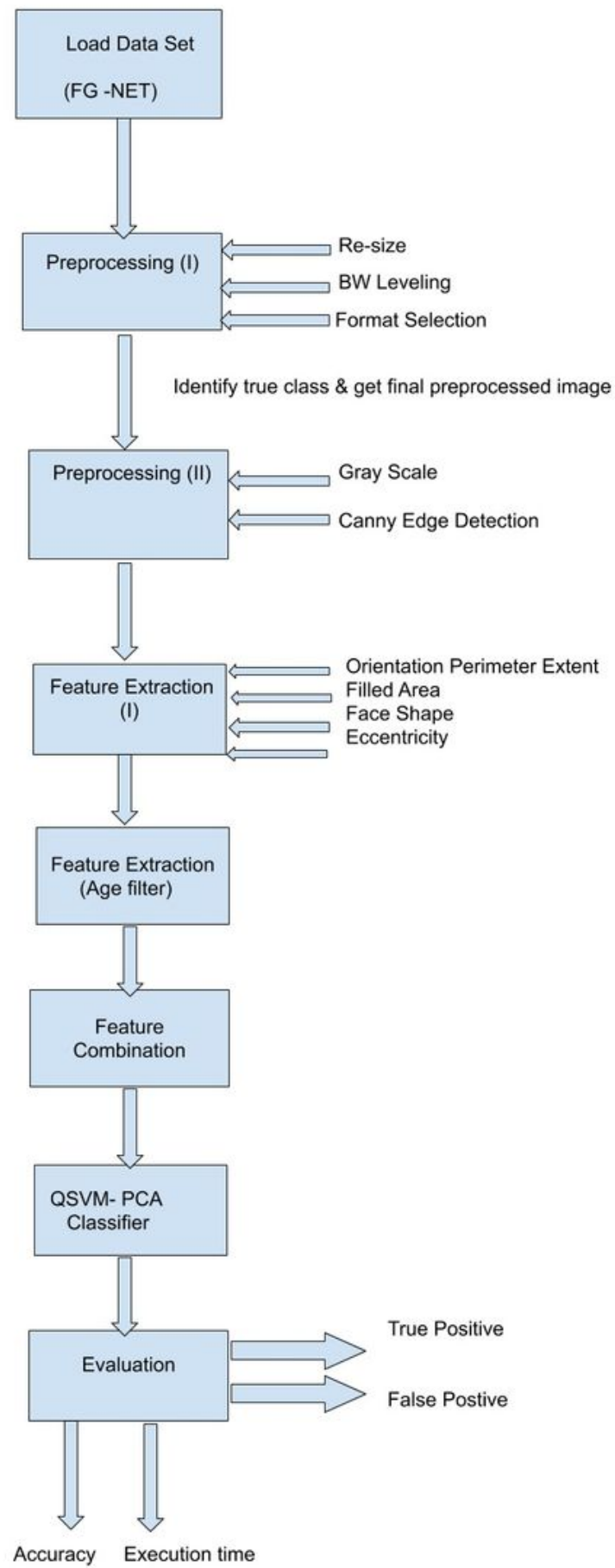

Figure 1

Demonstrated, Methodology 


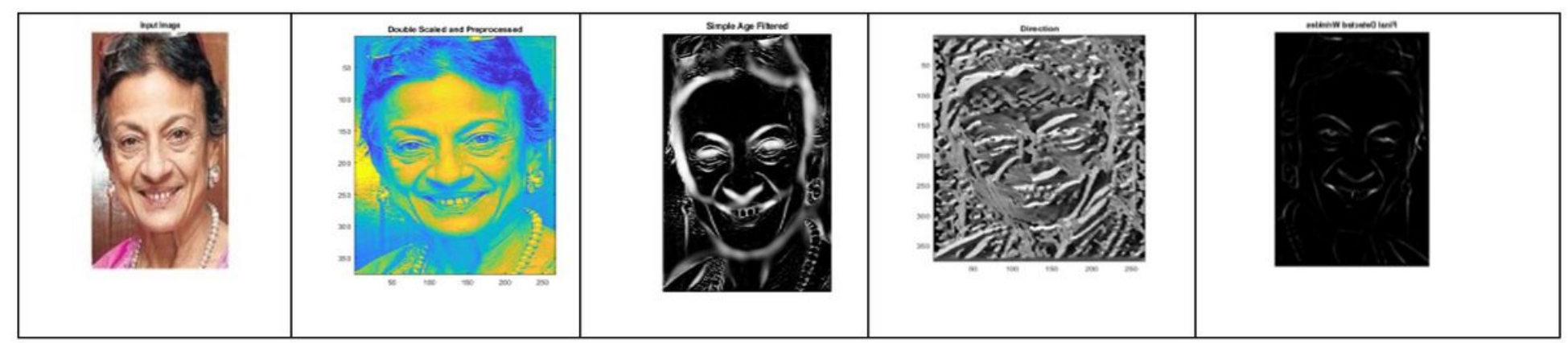

Figure 2

Sample image of FGNET dataset. 


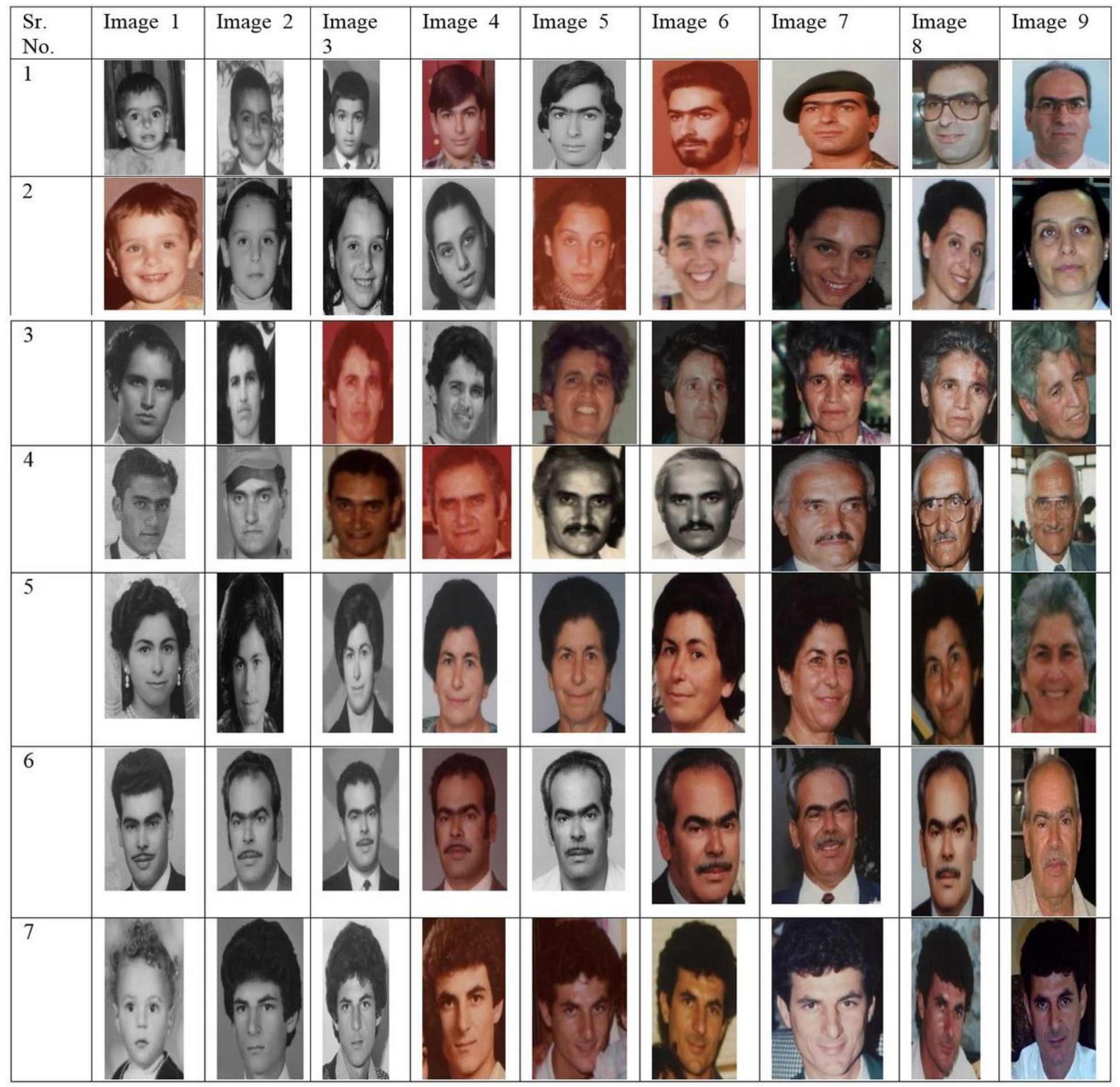

Figure 3

Sample images from FGNET database. 
True Positive Rates

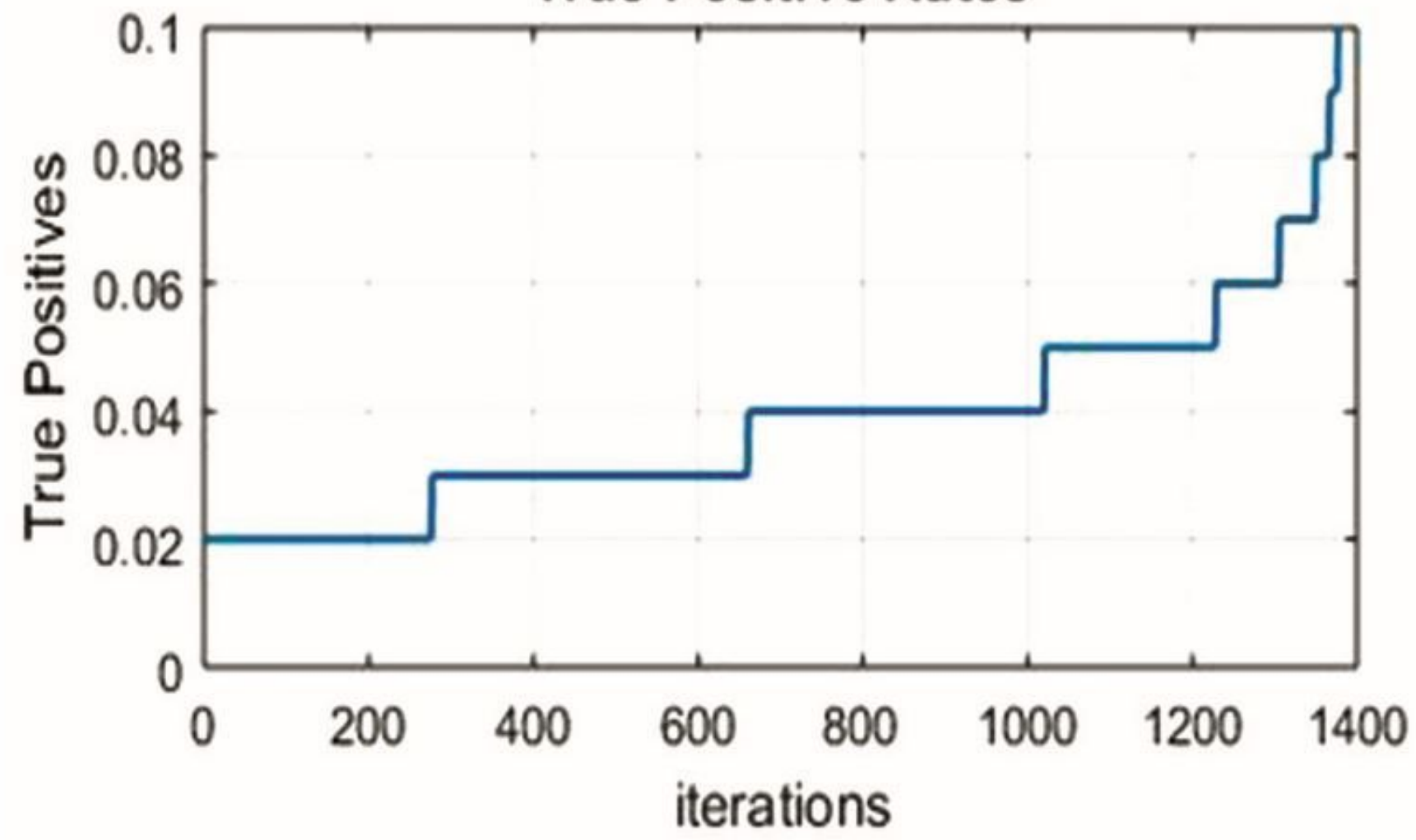

Figure 4

True PR (PR)

False Positive Rates

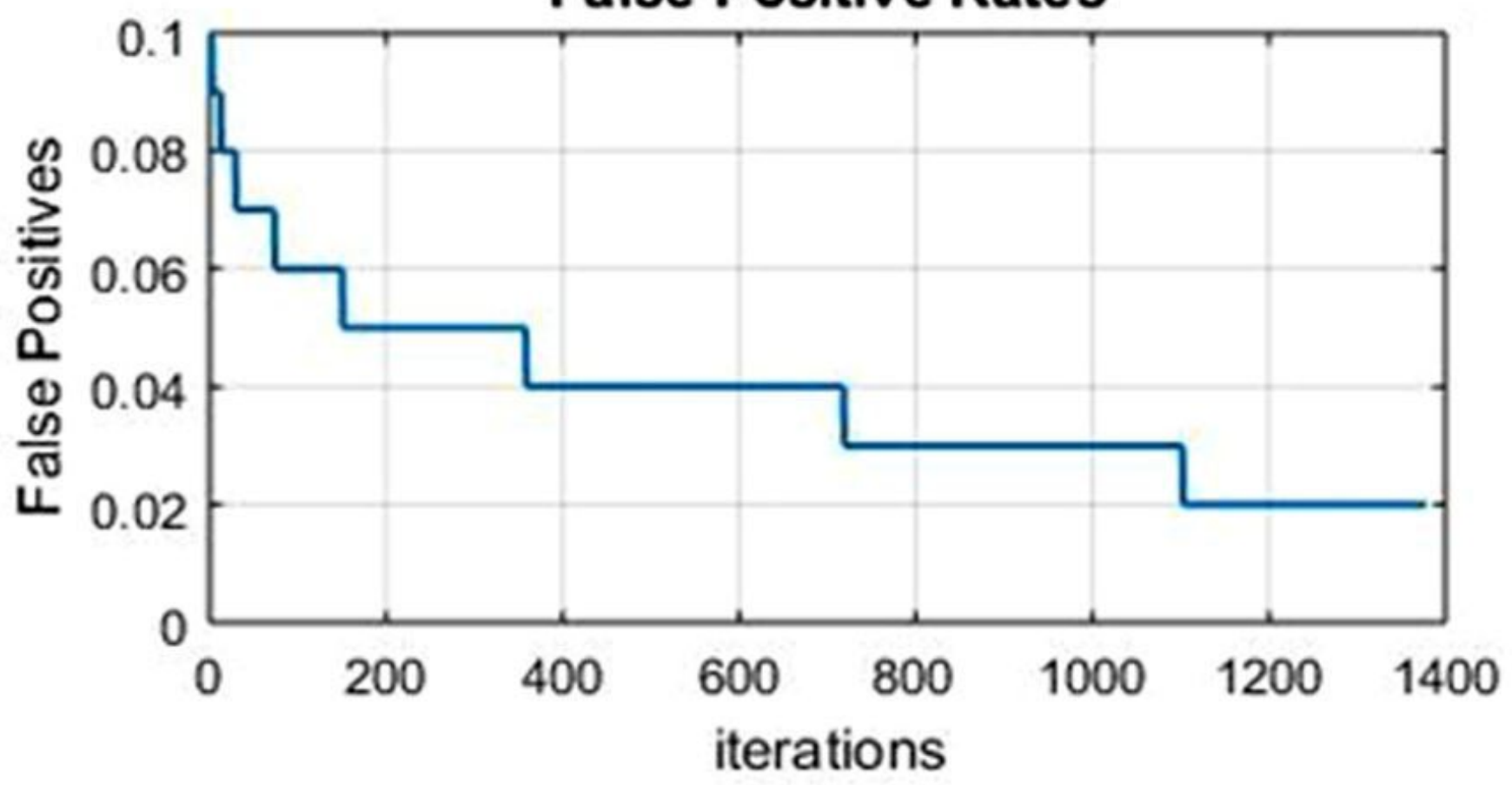


Figure 5

False PR

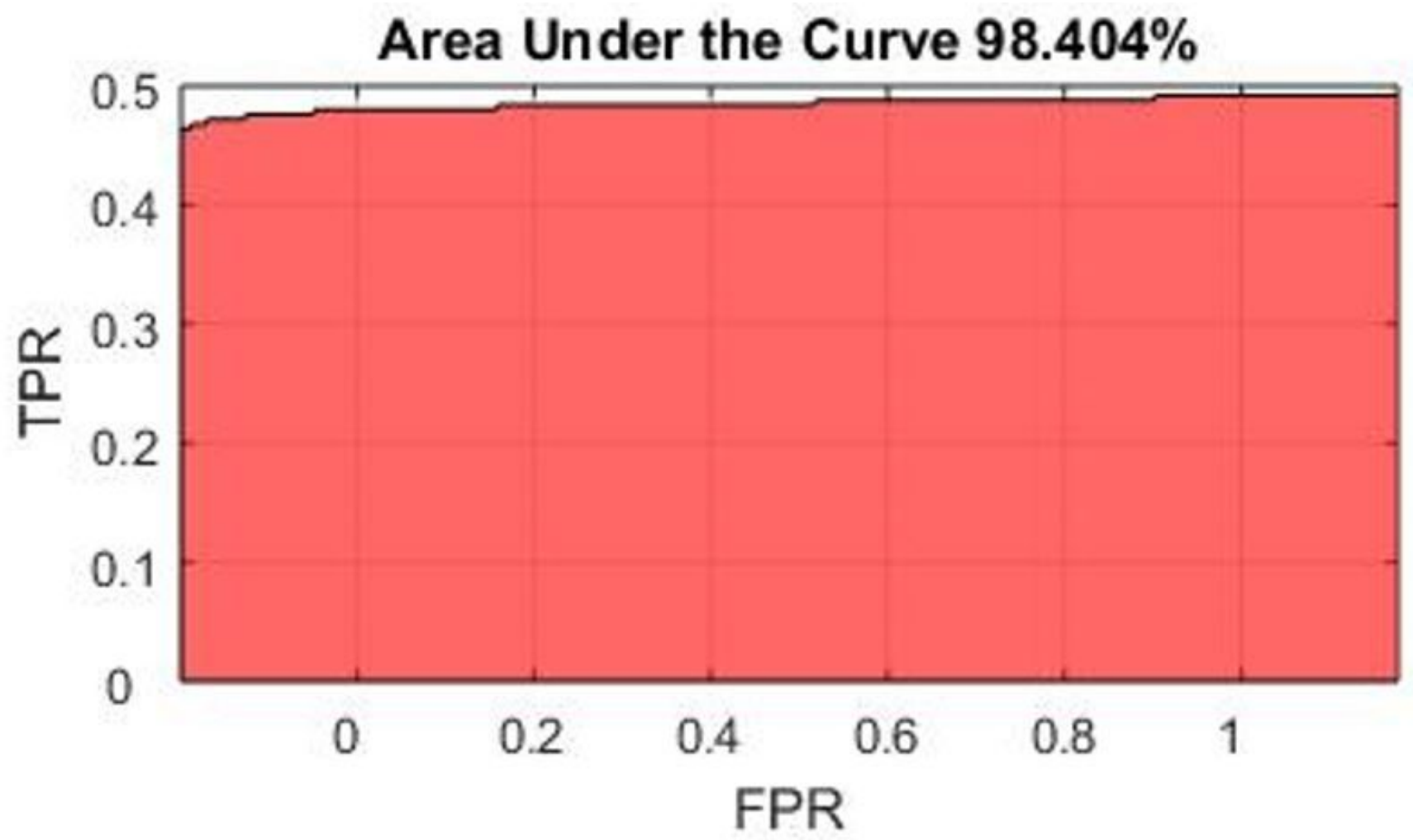

Figure 6

Comparison of True Positive \& False Positive 


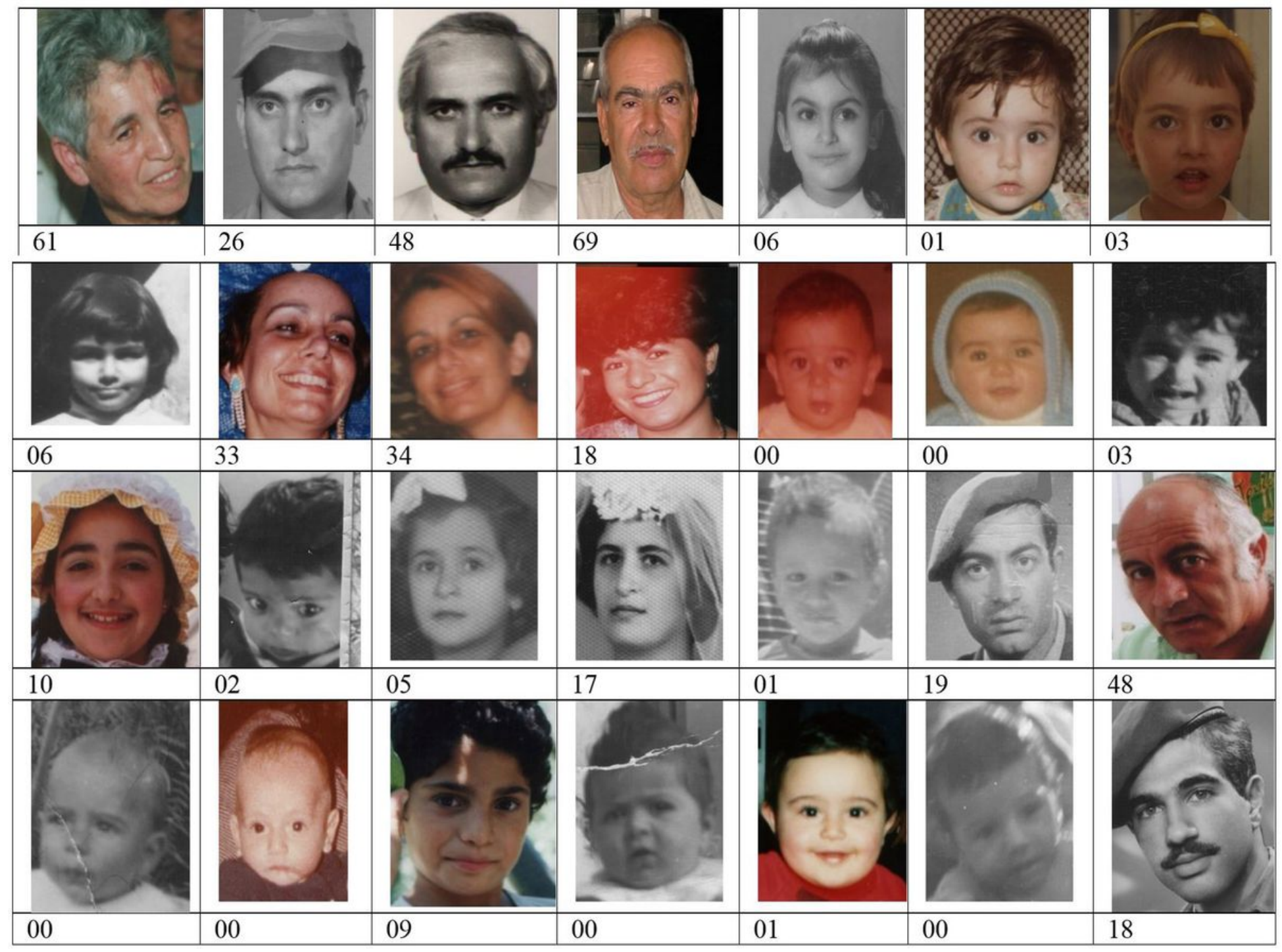

Figure 7

Some findings were incorrectly defined by the proposed FGNET dataset process. The numbers below display the age of the person. 